\title{
Relative Entropy and Efficiency Measure for diffusion-mediated transport processes
}

\author{
Roger Filliger* and Max-Olivier Hongler ${ }^{\dagger}$ \\ Institut de Production et Robotique (IPR), Laboratoire de Production Microtechnique (LPM), \\ Ecole Polytechnique Fédérale de Lausanne (EPFL), CH-1015 LAUSANNE.
}

(Dated: July 3, 2004)

\begin{abstract}
We propose an efficiency measure for diffusion-mediated transport processes including molecularscale engines such as Brownian motors (BMot) moving in ratchet potentials acting as mechanical "rectifiers". The efficiency measure is based on the concept of "minimal energy required to complete a task" and is defined via a class of stochastic optimal control problems. The underlying objective function depends on both the external force field (i.e. the fluctuation rectifier in the case of BMot) and the amplitude of the environmental noise. Ultimately, the efficiency measure can be directly interpreted as the relative entropy between two probability distributions, namely: the distribution of the particles in presence of the external rectifying force field and a reference distribution describing the behavior in absence of the rectifier.
\end{abstract}

PACS numbers: 05.70.-a, 05.40.-a, 05.10.Gg

\section{INTRODUCTION}

Despite to an already vast available literature, the fact that micro-particles immersed in a noisy environment can be transported by an ad-hoc rectification of the fluctuations, continue to attract attention directed towards applications [1]. The operations of these diffusion-mediated devices which essentially act as mechanical diodes, require basically $i$ ) a fluctuating environment and $i i$ ) a fluctuation rectifier which is driven by an external energy input. This mechanism is able to generate a net particles current which can be sustained even in the presence of an opposing force (i.e. an external load). It is therefore possible to extract a net useful work from these devices, a property appealing for applications in the molecular and microscopic size ranges. The possibility to extract work legitimates to use the word motors and also suggests that a suitable efficiency measure, namely the motor efficiency (ME), should be defined for these devices this is the goal of the present contribution.

Yet, the issue of efficiency of molecular motors has been and remains an important topic of its own (see e.g., [1] Chapt.6.9 and $[2,3])$. The main difficulty here is that on the microscopic scale the fluctuations do, simultaneously, favor and hinder the transport process. The external energy injected into the system is indeed dissipated via two mechanisms, $i$ ) the driving energy itself responsible for the transport process and $i i$ ) the heating of the medium which hinders the directed transport thanks to the fluctuation-dissipation relation.

In view of the conjugate actions played by the fluctuations, the study of energetics requires a precise formu-

*partially supported by the "FNSR" (Fonds National Suisse pour la Recherche).; Electronic address: roger.filliger@epfl.ch

$\dagger$ Also at FCT Portugal, CCM Universidade da Madeira.; Electronic address: max.hongler@epfl.ch lation of what the energy output of the system really is. Here we essentially adopt the point of view of I. Dérenyi et al. [4] which identifies the energy output with the minimum energy input $E_{i n}^{\text {min }}$ required to accomplish the same task as the molecular motor. Following [4], the generalized efficiency $\eta$ is defined as:

$$
\eta=E_{i n}^{\min } / E_{i n}
$$

where $E_{i n}$ is the external energy input.

The aim of this paper is to derive a new systematic method, to characterize the efficiency of the molecular motor. This will be achieved via a stochastic optimal control approach in which the objective function combines the conjugate actions of transport and dissipation. Formally, assume that a molecular motor operates according to the Langevin dynamics:

$$
\gamma \mathrm{d} X_{t}=\left(-V^{\prime}\left(X_{t}, t\right)-F\right) \mathrm{d} t+\sigma\left(X_{t}, t\right) d B_{t},
$$

which describes a Brownian particle in an overdamped regime (i.e. inertia is neglected). The stochastic equation is interpreted in the Stratonovich sense (see [5] for an account of Itô-Stratonovich modelling in the context of molecular motors) and models the so-called Brownian motor (BMot). Here $d B_{t}$ stands for $1 D$ standard Brownian motion, $\sigma(x, t)=\sqrt{2 \gamma k_{B} T(x, t)}$ controls the diffusion process with $k_{B}$ being Boltzmann's constant, $T(x, t)$ the absolute temperature field and $\gamma$ is the viscous friction coefficient. $F$ is a constant force modelling an external load and $V(x, t)$ is the "ratchet" potential through which the fluctuations are "rectified".

The net effect of the rectifying force given by $-V^{\prime}\left(X_{t}, t\right)$ is to drive an initial probability distribution $\delta_{x_{0}}$ at time $t=0$ to a final distribution $\mu_{\tau}$ at time $\tau>0$. In practice, there is a great freedom in choosing rectifying forces able to complete this task. Hence the natural idea behind the efficiency concept is to compare $-V^{\prime}(x, t)$ to an optimal drift field $u(x, t)$ which operates a similar task (namely 
transporting $\delta_{x_{0}}$ to $\mu_{\tau}$ within a time $\tau$ ) and simultaneously minimizes the objective functional:

$$
u \mapsto J_{\tau}(u)=\frac{1}{2} \mathbb{E}_{x_{0}} \int_{0}^{\tau}\left(\frac{u\left(X_{t}^{u}, t\right)}{\sigma\left(X_{t}^{u}, t\right)}\right)^{2} \mathrm{~d} t,
$$

where $X_{t}^{u}$ is evolving as:

$$
\gamma \mathrm{d} X_{t}^{u}=\left(u\left(X_{t}^{u}, t\right)-F\right) \mathrm{d} t+\sigma\left(X_{t}^{u}, t\right) d B_{t} .
$$

Note that the objective functional (sometimes called cost functional) depends on the stochastic dynamics via both the drift $u$ and the diffusion $\sigma$ fields. Due to the structure of the cost functional, transport with small diffusion is very costly - a property which reflects the diffusionmediated transport processes where fluctuations directly participate to the driving of particles. Define now the optimal rectifier $u^{*}(x, t)$ force field to be the one, able to achieve the transporting task, namely $\delta_{x_{0}} \mapsto \mu_{\tau}$ while minimizing the global value function $J_{\tau}\left(u^{*}\right)$ given by Eq. (3). With such an optimal rectifier at hand, the generalized efficiency of a BMot subject to a potential $V$ satisfying $0<J_{\tau}\left(V^{\prime}\right)<\infty$, is now simply defined as:

$$
\eta(\tau)=\frac{J_{\tau}\left(u^{*}\right)}{J_{\tau}\left(V^{\prime}\right)} .
$$

The ME $\eta(\tau)$ compares the expected costs incurred by both the optimal trajectories $X_{t}^{u^{*}}$ and the actual motor trajectories $X_{t}$ and therefore takes fully into account the probabilistic nature of the underlying process. This is, we believe, the conceptual advantage of the present approach over the one proposed in [4] where the minimum energy input is defined via a purely mechanical view not explicitly invoking the underlying stochastic dynamics.

From a mathematical point of view, the efficiency parameter given by Eq. (5) is well defined. It directly relies on the possibility to assign to given initial and final distributions (here $\delta_{x_{0}}$ and $\mu_{\tau}$ ) a unique Markov process - a problem first formulated by Schrödinger in [6]. A mathematically rigorous exposition of this program is given by P. Dai Pra [7]. The optimal control interpretation of the stochastic dynamics is thereby realized by the concept of logarithmic transformations introduced by Jamisson [8] and Flemming [9]. Using this approach, the minimal value $J_{\tau}\left(u^{*}\right)$ - entering the definition of $\eta(\tau)$ - coincides with the relative entropy $K\left(\mu_{\tau}, \bar{\mu}_{\tau}\right)$ between the distributions $\mu_{\tau}$ and $\bar{\mu}_{\tau}$ with $\bar{\mu}_{\tau}$ being the probability distribution at time $\tau$ describing the position $\bar{X}_{\tau}$ of a particle evolving under the control free dynamics

$$
\gamma \mathrm{d} \bar{X}_{t}=-F \mathrm{~d} t+\sigma\left(\bar{X}_{t}, t\right) \mathrm{d} B_{t}, \quad X_{0}=x_{0} \in \mathbb{R} .
$$

The efficiency of the Brownian motor then reads as

$$
\eta(\tau)=K\left(\mu_{\tau}, \bar{\mu}_{\tau}\right) / J_{\tau}\left(V^{\prime}\right) .
$$

This way of writing the ME, which is equivalent to Eq. (5), does not explicitly use the underlying control and is therefore intrinsic. Its connection with the control problem relies on the remarkable property that both: i) the quasi-free evolution, i.e. the evolution in absence of an external potential given by Eq. (6) and

ii) the evolution under the optimal control $u^{*}$

produce identical families of "most probable path" [7]. The concept of "most probable path" is based on a variational principle applicable for Langevin equations driven by White Gaussian Noise. The associated transition probability measures (i.e. the solutions of the relevant Fokker-Planck equations) can be expressed as the weighted sum over random trajectories with given initial and final conditions. The relative weights entering into the summation directly depend upon the drift and the diffusion coefficients and are expressed by the OnsagerMachlup functional [10]. Intuition suggests that the more probable a particular trajectory is, the more effectively it will contribute to the global value function. The fact that the most probable path under $u^{*}$ coincides with the most probable path under the quasi-free dynamics physically shows that the optimal control $u^{*}$ does in fact interfere as little as possible with the most probable trajectory of the quasi-free dynamics.

The paper is organized as follows. In section 2, we specify the hypothesis of the BM-model, state the optimal control problem and recall its solution. In section 3 we calculate the efficiency for a generic class of examples and indicate how to construct optimal rectifiers.

\section{PROBLEM FORMULATION}

Consider the controlled diffusion process $X^{u}(t)$ defined on some probability space $(\Omega, \mathcal{F}, \mathbb{P})$ that solves the following Stratonovich stochastic differential equation:

$$
\gamma \mathrm{d} X_{t}^{u}=\left(u\left(X_{t}^{u}, t\right)-F\right) \mathrm{d} t+\sigma\left(X_{t}^{u}, t\right) \mathrm{d} B_{t} .
$$

Formally, the above Langevin equation is obtained by replacing in Eq. (2) the force field $-V^{\prime}(x, t)$ by a control $u(x, t)$. The efficiency measure to be constructed relies on the following optimal control problem:

Problem $(P)$. Find an admissible control $u^{*}(x, t)$ such that:

(1) $X_{0}^{u^{*}}$ is distributed according to $\delta_{x_{0}}$ and $X_{\tau}^{u^{*}}$ according to $\mu_{\tau}$,

(2) between all admissible controls satisfying (1), the control $u^{*}$ minimizes the energy cost functional:

$$
u \mapsto J_{\tau}(u)=\frac{1}{2} \mathbb{E} \int_{0}^{\tau}\left(\frac{u\left(X_{t}^{u}, t\right)}{\sigma\left(X_{t}^{u}, t\right)}\right)^{2} \mathrm{~d} t .
$$

\section{Remarks.}

a) In order for Eq. (8) to admit a unique, $t$-continuous strong solution $X^{u}(t), \sigma$ and $u$ must satisfy the following classical assumptions: $\sigma$ and $u$ are linearly bounded in $x$ uniformly for $t \in[0, \tau]$ and 
satisfy a Lipschitz condition in $x$ for every fixed $t \in[0, \tau]$. These conditions ensure the uniqueness of $X_{t}^{u}$ and are supposed to hold from now on. In the autonomous case i.e., when both $u$ and $\sigma$ do not explicitly depend on time, existence and uniqueness of the solution are already guaranteed for $u, \sigma \in \mathcal{C}^{1}(\mathbb{R})$ [11].

b) A control $u\left(X_{t}(\cdot), t\right): \Omega \rightarrow \mathbb{R}$ is admissible if: $(i) u$ satisfies the regularity conditions stated in a), (ii) $u$ is adapted to the filtration $\mathcal{F}_{t}=\sigma\left\{X_{t}^{u}\right\}$ and $(i i i)$ $J_{\tau}(u)<\infty$. The potential $V$ entering into Eq. (2) is supposed to be admissible and non trivial i.e. $J_{\tau}\left(V^{\prime}\right)>0$.

c) A similar approach also holds for non-deterministic initial distributions $\mu_{0}$. The only restriction on $\mu_{0}$ is the existence of a finite second moment, i.e. $\int x^{2} \mu_{0}(\mathrm{~d} x)<\infty$. In this case, the problem $(\mathrm{P})$ does still have a well defined solution.

d) The Stratonovich stochastic differential equation (8) - commonly encountered in physical modelling where the White Gaussian Noise is viewed as the limit of shortly correlated colored noises - is equivalent to the Itô stochastic differential equation:

$\gamma \mathrm{d} X_{t}^{u}=\left[u\left(X_{t}^{u}, t\right)-F+\frac{1}{2} \sigma^{\prime}\left(X_{t}^{u}, t\right) \sigma\left(X_{t}^{u}, t\right)\right] \mathrm{d} t+\sigma\left(X_{t}^{u}, t\right) d B_{t}$

which, due to the fact that the noise term is a martingale, is more frequently used in control theory.

For $0 \leq s<t \leq \tau$ and $y, x \in \mathbb{R}$, denote by $q(s, y, t, x)$ the fundamental solution to the backward Fokker-Planck equation of the Markov process $\bar{X}_{t}$ defined in Eq. (6):

$$
\begin{aligned}
\frac{\partial}{\partial t} q(s, y, t, x) & =L q(s, y, t, x), \quad 0 \leq s<t \leq \tau \\
q(s, y, s, x) & =\delta_{x, y}
\end{aligned}
$$

where the operator $L(\cdot)$ is:

$$
L(\cdot)=\left[-F+\frac{1}{2} \sigma^{\prime}(x, t) \sigma(x, t)\right] \frac{\partial(\cdot)}{\partial x}+\frac{1}{2} \sigma(x, t)^{2} \frac{\partial^{2}(\cdot)}{\partial x \partial x}
$$

For $\mu$ and $\nu$, two probability distributions on the real line, write $\mathrm{d} \nu / \mathrm{d} \mu$ for the Radon-Nykodim derivative of $\nu$ with respect to $\mu$ (i.e. $\nu$ is assumed to be absolutely continuous with respect to $\mu$ ). Introduce now the relative entropy (also known as the Kullback "distance" [12]) $K(\mu, \nu)$ which quantifies the "discrepancy" between $\mu$ and $\nu$ :

$$
K(\mu, \nu)=\int_{\mathbb{R}} \log \left(\frac{\mathrm{d} \mu}{\mathrm{d} \nu}\right) \mathrm{d} \mu .
$$

The "distance" $K(\cdot, \cdot)$ is a commonly used for fitting and classifying statistical models, hypothesis testing and risk minimization. In the context of our minimization problem $(P)$, we have the following central result (Theorem 3.1 in $[7])$ :
Theorem. Suppose that $K\left(\mu_{\tau}, \bar{\mu}_{\tau}\right)<\infty$ and define $h: \mathbb{R} \times[0, \tau] \rightarrow \mathbb{R}$ by

$$
h(x, t)=\int_{\mathbb{R}} q(t, x, \tau, z) \frac{\mathrm{d} \mu_{\tau}}{\mathrm{d} \bar{\mu}_{\tau}}(z) \mathrm{d} z .
$$

Then

a) $u^{*}(x, t)=\sigma^{2}(x, t) \frac{\partial}{\partial x} \log (h(x, t))$ solves the control problem $(P)$ and

b) $J_{\tau}\left(u^{*}\right)=K\left(\mu_{\tau}, \bar{\mu}_{\tau}\right)$.

This result has three implications of practical relevance for the transport processes under study, namely:

1) It establishes that the $\mathrm{ME} \eta(\tau)$ takes values between zero and one (remember that $J_{\tau}(u)$ is positive). Moreover, $\eta(\tau)$ takes its maximum value 1 exactly if the motor realizes its task $\left(\delta_{x_{0}} \rightarrow \mu_{\tau}\right)$ in the energetically most favorable way.

2) It allows an intrinsic (i.e. control free) definition of the efficiency:

$$
\eta(\tau)=K\left(\mu_{\tau}, \bar{\mu}_{\tau}\right) / J_{\tau}\left(V^{\prime}\right),
$$

which relates the relative entropy between the distribution of the particle subject to $V$ and the distribution of the quasi-free particle with the expected cumulated costs $J_{\tau}\left(V^{\prime}\right)$.

3) It furnishes an explicit formula for $u^{*}$ which can be used as a theoretical guideline to physically realize optimal motors.

\section{APPLICATIONS FOR A GENERIC CLASS OF BROWNIAN MOTORS}

In the couple of examples which follow, we suppose that the Brownian particle is released at time $t=0$ from $X_{0}=0$ and evolves under the isothermal dynamics:

$$
\gamma \mathrm{d} X_{t}=\left(-V^{\prime}\left(X_{t}\right)-F\right) \mathrm{d} t+\sqrt{2 D_{0}} d B_{t},
$$

with $D_{0}=\frac{k_{B} T}{\gamma}$ constant. The next example 3.1 deals with a the potential $V(x)$ - periodic in $x$ with period $L$. We calculate $\eta$ in the central limit regime. In example 3.2 , we impose the optimal efficiency $\eta=1$ (i.e. the BMot operates under optimal conditions) and derive the associated optimal force field.

\section{A. The efficiency for Brownian Motors in the central limit regime}

Consider now a Brownian particle on a coarse grained space-scale $(\gg L)$ such that the evolution can be approximately viewed as the succession of single, independent 
and identically distributed hopping events ([1] p.88). Invoking the central limit theorem, the distribution of the particle for large $\tau$ approaches a Gaussian measure $\mu_{\tau}$ with density:

$$
f(x, \tau)=\frac{1}{\sqrt{4 \pi D_{e f f} \tau}} \exp \left(-\frac{\left[(a \tau-x)^{2}\right]}{4 D_{e f f} \tau}\right) .
$$

Here, $a=\lim _{t \rightarrow \infty} X_{t} / t$ is the average particle current and $D_{\text {eff }}=\lim _{t \rightarrow \infty}\left(\left\langle X_{t}^{2}\right\rangle-\left\langle X_{t}\right\rangle^{2}\right) /(2 t)$ is the effective diffusion coefficient. These two basic observables - measurable in actual applications [13] - are analytically related to the modelling parameters entering into Eq. (16) through [14]:

$$
\begin{aligned}
a & =D_{0} \frac{1-e^{-L F / k_{B} T}}{\frac{1}{L} \int_{0}^{L} \mathrm{~d} x I_{+}(x)}, \\
D_{e f f} & =D_{0} \frac{\frac{1}{L} \int_{0}^{L} \mathrm{~d} x I_{+}(x)^{2} I_{-}(x)}{\left(\frac{1}{L} \int_{0}^{L} \mathrm{~d} x I_{+}(x)\right)^{3}}
\end{aligned}
$$

with

$$
I_{ \pm}(x)=\int_{0}^{L} \mathrm{~d} y \exp ( \pm V(x) \mp V(x \mp y)-y F) /\left(k_{B} T\right) .
$$

According to Eq. (6), the quasi-free particle evolves under the dynamics:

$$
\bar{X}_{t}=-\frac{F}{\gamma} t+\sqrt{2 D_{0}} B_{t}
$$

Its distribution $\bar{\mu}_{\tau}$ at $\tau$ is therefore a Gaussian measure with density:

$$
\bar{f}(x, \tau)=\frac{1}{\sqrt{4 \pi D_{0} \tau}} \exp \left(-\frac{\left[x-\frac{F}{\gamma} \tau\right]^{2}}{4 D_{0} \tau}\right) .
$$

The relative entropy of the two Gaussian measures Eqs. (17) and (22) reads as:

$$
\begin{aligned}
K\left(\mu_{\tau}, \overline{\mu_{\tau}}\right)= & \int_{\mathbb{R}} \ln (f(x, \tau) / \bar{f}(x, \tau)) f(x, \tau) \mathrm{d} x \\
= & \frac{(F / \gamma-a)^{2}}{4 D_{0}} \tau+ \\
& \left\{\frac{1}{2} \ln \left(\frac{D_{0}}{D_{\text {eff }}}\right)+\frac{D_{\text {eff }}-D_{0}}{2 D_{0}}\right\} .
\end{aligned}
$$

This expression, valid for large $\tau$, enables to separately appreciate the influence of the potential $V$ on the particles current and on the rectifying process. The first term describes the external energy input necessary to maintain the particles current $a$. The symmetry in the difference $F / \gamma-a$ shows that exceeding or undergoing the quasi-free current $F / \gamma$ by the amount $|F / \gamma-a|$ requires the same energy input. The second contribution - non-negative as the former one - is related to the part of the energy input required to get an effective diffusion $D_{\text {eff }}$. In contrast to the current difference $F / \gamma-a$, the diffusion difference
$D_{0}-D_{\text {eff }}$ contributes non-symmetrically to the relative entropy. Setting:

$$
f\left(D_{e f f}\right):=\ln \left(\frac{D_{0}}{D_{e f f}}\right)+\frac{D_{e f f}-D_{0}}{D_{0}},
$$

we indeed have:

$$
f\left(D_{0}-\Delta\right)>f\left(D_{0}+\Delta\right), \quad \Delta \in\left(0, D_{0}\right) .
$$

Hence, rectifying the diffusion to ensure that $D_{\text {eff }}=$ $D_{0}-\Delta$ with $\Delta \geq 0$ costs more than to let it increase by the same amount (i.e. $D_{\text {eff }}=D_{0}+\Delta$ ). In the limiting case where $V$ "ties down" the effective diffusion to zero, we have $f(0)=\infty$ and consequently, the relative entropy $K\left(\mu_{\tau}, \overline{\mu_{\tau}}\right)$ becomes infinite.

On the other hand, the cost functional for the given potential is:

$$
\begin{aligned}
J_{\tau}\left(V^{\prime}\right) & =\frac{1}{4 \gamma k_{B} T_{0}} \mathbb{E} \int_{0}^{\tau} V^{\prime}\left(X_{t}\right)^{2} \mathrm{~d} t \\
& =\frac{\tau}{4 \gamma^{2} D_{0}} \mathbb{E} \frac{1}{\tau} \int_{0}^{\tau} V^{\prime}\left(X_{t}\right)^{2} \mathrm{~d} t
\end{aligned}
$$

which due to ergodicity behaves asymptotically as:

$$
J_{\tau}\left(V^{\prime}\right) \simeq \frac{\tau}{4 \gamma^{2} D_{0}}\left\langle V^{\prime 2}\right\rangle .
$$

Here the ensemble mean $\left\langle V^{\prime 2}\right\rangle$ is taken over one space period of $V$. Using Eqs. (23) and (27), we have:

$$
\eta(\tau)=\frac{K\left(\mu_{\tau}, \bar{\mu}_{\tau}\right)}{J_{\tau}\left(V^{\prime}\right)}=\frac{(F-\gamma a)^{2}}{\left\langle V^{\prime 2}\right\rangle}+\mathcal{O}\left(\frac{1}{\tau}\right)
$$

where explicitly the $\mathcal{O}\left(\frac{1}{\tau}\right)$ term is given by:

$$
\mathcal{O}\left(\frac{1}{\tau}\right)=\frac{1}{\tau} \frac{2 \gamma^{2} D_{e f f}}{\left\langle V^{\prime 2}\right\rangle}\left(1+\frac{D_{0}}{D_{e f f}}\left[\ln \left(\frac{D_{0}}{D_{e f f}}\right)-1\right]\right)
$$

In the central limit regime, reached for $\tau \rightarrow \infty$, only the part contributing to the transport process influences the efficiency measure, namely:

$$
\eta(\tau)=\frac{(F-\gamma a)^{2}}{\left\langle V^{\prime 2}\right\rangle}, \text { for } \tau \rightarrow \infty .
$$

The parameters $F, \gamma, V^{\prime}, D_{0}$ and $D_{\text {eff }}$ entering into Eqs. (29) and (30) are experimentally measurable as it is discussed in [13]. Note in addition that the time series of motor positions, measurable with the current bioengineering experimental technology, allow to solve a reverse problem, namely to reconstruct the rectifying potential $V$ [15]. Hence, the efficiency $\eta(\tau)$ is clearly not a mere theoretical concept but is available for actual applications.

\section{B. Conceiving efficient Brownian Motors.}

As stated by Derényi et al. in [4], it is only a matter of time before the first man-made molecular motors will be 
manufactured. One therefore is potentially interested in building highly efficient transport motors. Starting from the quasi-free dynamics:

$$
\mathrm{d} \bar{X}_{t}=-\frac{F}{\gamma} \mathrm{d} t+\sqrt{2 D_{0}} \mathrm{~d} B_{t}
$$

with $F, \gamma$ and $D_{0}$ constant, we now construct an optimal force field $u^{*}(x, t)$. The fundamental solution to the associated backward evolution equation Eq. (12) is:

$q(s, y, t, x)=\frac{1}{\sqrt{4 \pi D_{0}(t-s)}} \exp \left(-\frac{\left[\left((x-y)-\frac{F}{\gamma}(t-s)\right)^{2}\right]}{2 D_{0}(t-s)}\right)$

and $\bar{\mu}_{\tau}$ is Gaussian with density $\bar{f}(x, \tau)=$ $\int_{\mathbb{R}} q(0, y, \tau, x) \delta_{0}(\mathrm{~d} y)=q(0,0, \tau, x)$. The minimum energy control $u^{*}(x, t)$ is now:

$$
u^{*}(x, t)=2 D_{0} \frac{\partial}{\partial x} \ln \left(\int_{\mathbb{R}} q(t, x, \tau, z) \frac{f(z, \tau)}{q(0,0, \tau, z)} \mathrm{d} z\right)
$$

which after tedious but elementary calculations yields:

$$
u^{*}(x, t)=\frac{D_{0}\left(\frac{F}{\gamma} t-x-T\left(a-\frac{F}{\gamma}\right)\right)}{D_{0}(\tau-t)+D_{e f f} \tau} .
$$

Setting $\frac{F}{\gamma}=a$ and $D_{e f f}=D_{0}$ and prolonging $u(x, t)$ periodically in $x$ resp. $t$ with period $L$ resp. $\tau$ to $\mathbb{R} \times \mathbb{R}_{+}$, we find the optimal drift field:

$$
\begin{aligned}
u^{*}(x, t) & =\frac{a t-x}{2 \tau-t}, \quad 0 \leq x \leq L, \quad 0 \leq t \leq \tau \\
u^{*}(x+L, t) & =u^{*}(x, t), \quad u^{*}(x, t+\tau)=u^{*}(x, t) .
\end{aligned}
$$

As it stands, $u^{*}(x, t)$ is linear in $x$ and depends explicitly on time. The time-dependent drift field $u^{*}(x, t)$ derives from the class of traveling potential ratchets (see e.g., [1] Chapt. 3.3 for a classification scheme for BMot) and might serve as a simple guideline in the attempts to actually realize isothermal molecular motors.

\section{CONCLUSIONS}

The stochastic optimal control formalism is well suited to define an efficiency measure for diffusion-mediated transport processes governed by the Langevin dynamics. The objective function to be minimized combines, in a single functional, the competing effects of the drift and diffusion forces responsible for the transport process. The explicit construction of the optimal force field that minimizes the objective functional, offers a rigorous and systematic way to conceive efficient diffusion-mediated transport devices.
[1] P. Reimann, Phys. Rep. 361, 57 (2002).

[2] J. Parrondo and B. D. Cisneros, Applied Physics A 75, 179 (2002).

[3] K. Sekimoto, Journal of the physical society of Japan 66(5), 1234 (1997).

[4] I. Derényi, M. Bier, and R. Astumian, Physical Review Letters 83 (5), 903 (1999).

[5] M. Matsuo and S. Sasa, Physica A 276, 188 (1999).

[6] E. Schrödinger, Phys. Math. 144 (1931).

[7] P. D. Pra, Applied mathematics and Optimization 23, 313 (1991).

[8] B. Jamison, Z. Wahrscheinlichkeitstheorie verw. Gebiete 32, 323 (1995).

[9] W. Flemming and H. Soner, Controlled Markov Processes and Viscosity Solutions (Springer-Verlag, 1993).
[10] D. Durr and A. Bach, Comm. Math. Phys. 60, 153 (1978).

[11] W. Horsthemke and R. Lefever, Noise-Induced Transitions. (Springer-Verlag, 1984).

[12] S. Kullback, Information theory and Statistics (Dover, New York, 1968).

[13] H. Wang, IEE Proc.-Nanobiotechnol. 150 (3), 127 (2003).

[14] P. Reimann, C. van den Broeck, H. Linke, P. Hänggi, M. Rubi, and A. Pérez-Madrid, Physical Review Letters 87, 010602 (2001).

[15] K. Visscher, M. Schnitzer, and S. Block, Nature 400, 184 (1999). 